\title{
Research on the model of the production of hot forming process and the optimization method of production cycle
}

\author{
Ju Meng, Liang Wang, Qiang Wang, Yi Sun, and Yisheng Zhang *
}

State Key Laboratory of Materials Processing and Die \& Mould Technology, Huazhong University of Science and Technology, Wuhan 430074, P.R. China

*zhangys@mail.hust.edu.cn

Keywords: hot stamping; low energy consumption; production flow; optimization.

Abstract. With the increasingly high demand for energy saving, the demand for lightweight vehicles emerged. Hot stamping of high strength steel is a very important part of automobile lightweight. How to establish an efficient, reliable, energy saving hot stamping production lines will be an integral part of automotive lightweight issues in the production process. Based on the SIGMA hot stamping production line, this paper uses a parallel, multi-scale analysis method and presents an analysis model of production cycle. According to the motion characteristics of industrial robots and transmission principle, the operation steps of industrial robots are mainly analyzed to improve the efficiency of collaborative work. The paper finds production bottlenecks, looks for optimization program to shorten cycle and optimize them, compares the old and new programs and completes the test of feasibility of it, thereby achieves the purpose of improving the efficiency of sheet delivery, shortening the production cycle, and improving production efficiency.

\section{Introduction}

Hot stamping is a positive method for strength enhancement and weight reduction. Compared with traditional stamping process, hot stamping process is accompanied by dramatic change of heat, and it consumes a lot of energy during heating.

This paper mentions 2 types of hot stamping line locally developed, hot stamping test line [1] and production line [2]. The test line consists of a box furnace for blank heating, a $2000 \mathrm{kN}$ electric servo press $[3,4]$ for stamping and quenching, a joint robot for blank transportation before and after heating up. The production line consists of 2 multi-chamber box furnace, 7 high-speed multi-axis cartesian robots, a $6000 \mathrm{kN}$ electric servo press. These 2 lines are fully digital-controlled and flexible. The servo press has enough ability to adjust holding cycle, stamping speed and motion according to process demands. The box furnace offers anti-oxidation atmosphere and heats blanks to temperature above $950 \square$. The joint robot in test line also coordinates operating order of the press and furnace. Cartesian robots in production line deliver blanks cooperatively and two of them are specially designed for door operation of the furnace. The local developed lines are energy-efficient and environment-friendly.
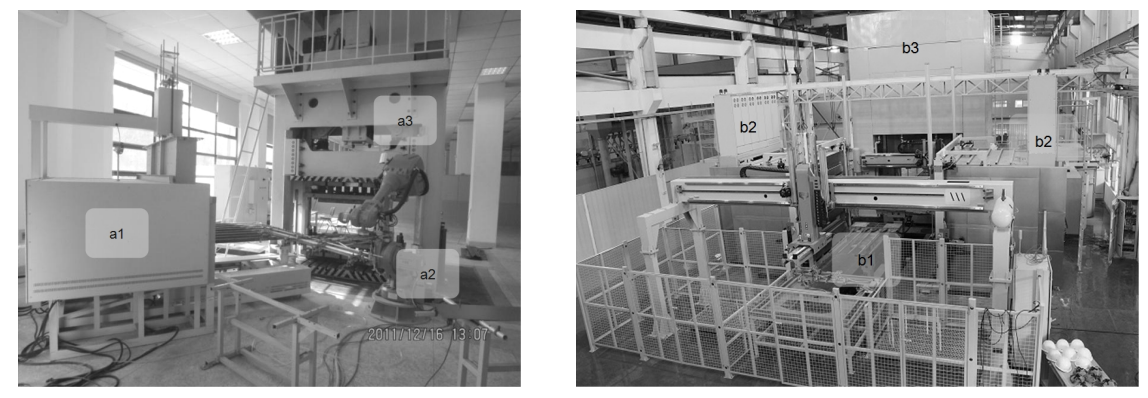

Fig. 1: The $2000 \mathrm{kN}$ hot stamping test line and SIGMA6000 hot stamping production line: a1) box furnace, a2) joint robot, a3) $2000 \mathrm{kN}$ electric servo mechanical press, b1) multi-axis cartesian robots, b2) multi-chamber furnace, b3) $6000 \mathrm{kN}$ electric servo mechanical press 


\section{Production Control Modeling of Hot Stamping Line}

Control Model of Hot Stamping Line. Hot stamping line consists of different equipment with various controller and its automation involves integration and interaction of controllers, control flow and information flow. These limitations require that control model has good compatibility with a wide range of adaptability.

The transportation process is obviously more complicated than heating or forming process. Robots should transport in designed time window and cooperate accurately with other robots and equipment. So transportation is the key of automation of this type of hot stamping line. Efficient cooperation of robots is the precondition for high efficient automation.

An improved RCS reference model [5] is presented for multi-equipment cooperation in this paper. The improved RCS model is a multi-layer and multi-resolution architecture consisting of compute nodes. At the lower layer, these elements generate goal-search-oriented reactive behavior, while at the higher layer, produce a goal-definition-oriented deliberate behavior. Through the grading process structure, interactions between the functional elements can produce behaviors like perception, cognition, imagination, reasoning, and so on. At the lower layer, the process area in time and space is small and the information processed is in a high resolution. But at the higher layer, the process area in time and space is wide and the information processed is in a low resolution. This approach enables that behaviors which need high accuracy and rapid response can be handled at a lower layer within a smaller space over a short period of time, while long-term planning and abstract concepts can form and can be realized at a higher layer within large spatiotemporal scope. This is the core of RCS of hierarchical intelligence.

According to improved RCS reference model, control system of hot stamping line can be divided into 2 layers: command layer and task layer. The command layer includes several command nodes and is responsible for task planning, process control, map creation and analysis. The task layer contains multiple task execution nodes and complete special tasks. According to different designs of production line, the number and function of node can be adjusted at each layer, but the overall structure remains the same. Communication between nodes is via industrial-grade links.

Analysis Model of Production Cycle and Optimization Method. Cycle of production line is the time consumed to produce one product. In theory, the minimal cycle of a production line is never less than the maximum cycle of any equipment in the line, as shown in Equation 1.

$$
\mathrm{C}_{\text {line }} \geq \max \left(\mathrm{C}_{\mathrm{e} 1}, \mathrm{C}_{\mathrm{e} 2}, \ldots, \mathrm{C}_{\mathrm{en}}\right) \text {. }
$$

Where, $\mathrm{C}_{\text {line }}$ is cycle of a line, $\mathrm{C}_{\mathrm{ei}}$ is cycle of equipment $\mathrm{i}$. It is assumed that there are $\mathrm{n}$ equipment in the line and $\mathrm{i}$ is from 1 to $\mathrm{n}$.

Cycle of a line is related with the cycle of equipment in line and affected by time consumption of equipment interlinking. In a production line, if there are 2 equipment whose running space overlaps or is directly related, there are interlinking time between these 2 equipment. So, cycle of a line always consist of cycle of an equipment, interlinking time with its related equipment, and waiting time, as shown in Equation 2.

$$
\mathrm{C}_{\text {line }}=\mathrm{C}_{\mathrm{ei}}+\mathrm{t}_{\mathrm{ri}}+\mathrm{t}_{\mathrm{wi}} \text {. }
$$

Where, $t_{r i}$ is interlinking time between equipment $i$ and its related equipment, $t_{w i}$ is waiting time of equipment i. Specially, when the line runs in the minimal cycle, the waiting time of the longest-cycle equipment is 0 .

So there are 2 strategies to reduce line cycle: the first is to reduce equipment cycle $\mathrm{C}_{\mathrm{e}}$, the second is to reduce interlinking time. Strategy 1 is closely related with characteristics of the equipment, which is not common. In strategy 2 , the optimization methods are usually motion cooperation of multiple equipment, key space quick dodge, motion path optimization and so on. 


\section{Implementation and Discussion}

Production Flow. In hot stamping production line, overall control system (OCS) is at the command layer. Transporting robots, furnaces and the press are at the task layer. The automation control architecture of this production line is a heterogeneous distributed system. Controllers of all equipment are different. All the equipment are independently controlled, loosely coupled with other nodes. Each equipment perceives status of the whole line or part of it through information exchange, and execute its own tasks when relative status are met.

The control flow of the production line is as follows. All the operations starts from status perception. OCS perceives status of all the nodes at the task layer, creates a map of the line and then analyses it. When the line status meet the requirement of next task, OCS send task information to related node at the task layer. The finite state machine is used here. Completely depending on status of task-related node at the task layer, OCS at the command layer makes decision about when to assign a task and whether a task has been executed or finished. The equipment node at the task layer determines whether to perform a task or not according to both instructions from the command layer and status of related equipment. OCS also has a module for security monitoring, which will ceaselessly check status information from all nodes in this system. When an exception occurs, in order to ensure the safety of equipment and operators, OCS will immediately turn to exception-handling procedure, obtain details, determine exception types, start handle exception by standard procedure, or terminate the whole line and report it to operator for troubleshooting. Figure 2 shows the control system of the hot stamping production line.

Industrial Ethernet is adopted as communication links in this production line, which makes it possible for massive detail information of equipment to be converged.

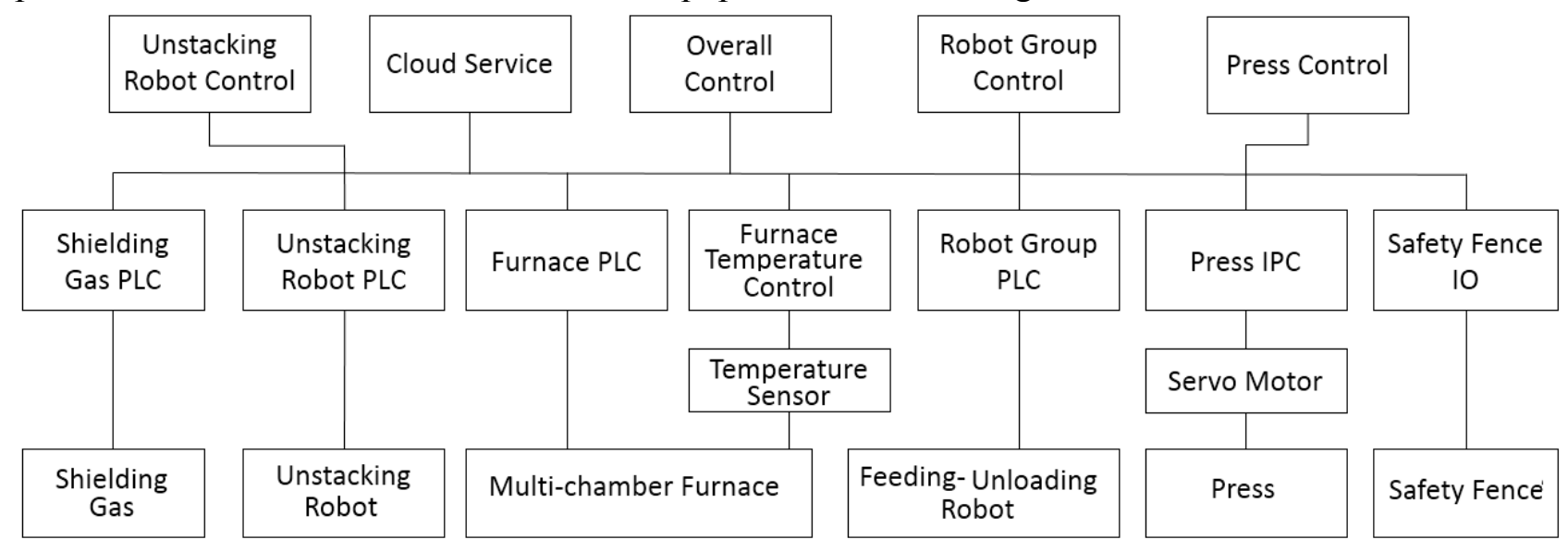

Fig. 2 Control system of SIGMA 6000 hot stamping production Line

Optimization of Production Cycle. Key of production cycle optimization is reduction of time consumed by equipment interlinking. Equipment-Interlinking Region (EIR) is a public space shared by equipment. EIR can be occupied by only one equipment at a certain time. Monopolization of EIR determines that if an equipment attempts to enter an occupied EIR, this occupied EIR must be released by occupying equipment. Figure 2 shows 7 EIRs in the SIGMA6000 hot stamping production line. Among them, region 1-5 is related to heating equipment, region 6-7 related to forming equipment.

A bottleneck cycle of the production line is the interlinking cycle including furnaces, the furnace-feeding robot (FFR) and the furnace unloading robot (FUR). It is because that FFR and FUR share a straight track along the direction of the line. The 2 robots in shared track should dodge region 4 in Figure 3 to compete assigned task. This means the interlinking procedure takes much more time. In order to reduce the interlinking time, the limited space must be fully made use of. The axes of these 2 robots along the specific trace are altered from coupling movement to non-coupling asynchronous movement. At the essential prerequisite of security, it is analyzed that how the 2 moving axes interfered in space. And finally this interlinking cycle is promoted by $50 \%$ through non-interference-of-space motion. 
Another important interlinking cycle is hot blank transporting, in which the press, press-feeding robot, press-unloading robot are involved. The promotion of this cycle is propitious to decrease aim temperature of heating, reduce energy consumption and lower cost.

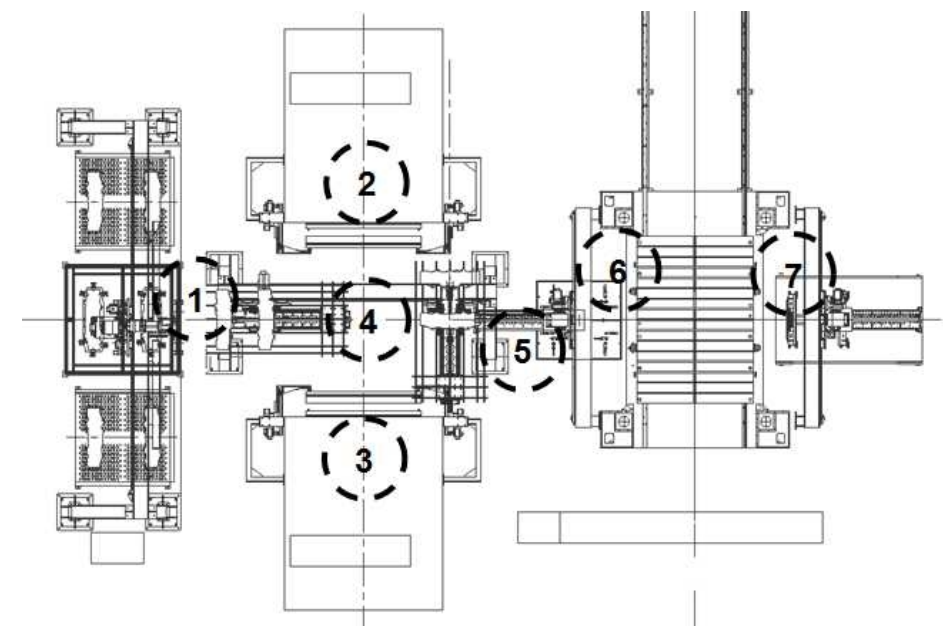

Fig. 3 Equipment-Interlinking Regions in SIGMA 6000 hot stamping production line.

\section{Summary}

This paper presents a hot stamping test line and a production line locally developed. Both lines adopt electrical mechanical linkage servo press and low-heat-capacity box furnace. Blank/part transporting tasks are performed by a joint robot in the test line and 7 high-speed multi-axis Cartesian robots in the production line. The lines are high energy efficiency and environment friendly. In the production line, some robots are specially designed, such as doors of furnaces are opened and closed by specified robots, FUR directly pass the hot blank on to PFR.

The control system of hot stamping production line is designed based on improved RCS reference model. The entire control architecture is reformed from traditional centralized control to distributed control, decoupled and layered. A model for cycle analysis is presented. Based on the model, cycle of production line is analyzed and its composition and impact factors are obtained. 2 optimization methods, motion cooperation of multiple equipment and key space quick dodge, are adopted.

\section{Acknowledgements}

This research work is financially supported by the National Natural Science Foundation of China (Grant No. 51275185 and No.51405171).

\section{References}

[1] Zheng Wang, Yisheng Zhang, Huiqiang Liu. Optimization of industrial robots transmission path in hot stamping production line, Forging \& Stamping Technology,37(2012),No.6,pp 31-34.

[2] Wang L, et al.: High efficiency and low energy consumption electric servo press for hot stamping of high strength steel. In: CAMS 2014. Nov. 25-27, 2014.

[3] Mo J. H., et al.: The Properties and Operation of Large Mechanical Linkage Servo Press. In: China Metal Forming Equipment \& Manufacturing Technology, (2009), No. 5, pp 35-40.

[4] Osakada K., et al.: Mechanical servo press technology for metal forming. In: CIRP Annals Manufacturing Technology 60 (2011), No. 2, pp 651-672.

[5] Information on http://www.isd.mel.nist.gov/projects/rcs/isam/ISAM_web.htm 